\section{Efficacy of portable $X$-ray in identifying retained suture needles in ophthalmologic cases}

\begin{abstract}
Aims To evaluate the role and efficacy of portable X-ray in reliably identifying retained suture needles in ophthalmologic surgical cases.

Methods Ophthalmologic 10-0 nylon suture needles were placed into $\mathbf{1 0}$ of 20 porcine eyes positioned within the orbit of a human skull. Portable, plain film radiographs were taken of all eyes with a C-arm fluoroscopy machine.

The films were reviewed by six ophthalmologists and six radiologists who were blinded to the presence or absence of needles, as well as the number of needles used in the protocol. The sensitivity and specificity of identifying retained suture needles by plain film radiographs were then analysed based on the results of the physicians' interpretations of the films.
\end{abstract}

Results The overall sensitivity and specificity for physicians reviewing radiographs with suspected intraoperative suture needles were 54 and $77 \%$, respectively. When looking separately at radiologists reviewing the films, the sensitivity was $57 \%$ and the specificity was $87 \%$. Ophthalmologists reviewing the films identified retained needles less reliably with a sensitivity of $51 \%$ and a specificity of $67 \%$.

Conclusions Significant controversy exists regarding the reliability and utility of plain film radiography in identifying intraocular foreign bodies. This study demonstrates that the use of plain film radiographs to rule-out the presence of an intraocular surgical needle is neither a sensitive nor specific imaging modality.

Eye (2009) 23, 1731-1734; doi:10.1038/eye.2008.320; published online 17 October 2008

JZ Kieval ${ }^{1,2}$, M Walsh $^{3}$, PA Legutko ${ }^{4}$ and MK Daly ${ }^{1,2}$

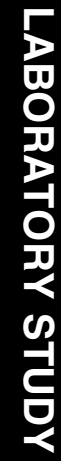

Keywords: radiograph; ocular foreign body; screening procedure

\section{Introduction}

Retained surgical needles after ophthalmic surgery pose a significant risk to patients' ocular health. Foremost is the concern for ocular morbidity, including globe perforation, inflammation, infection, or toxicity that may exist with an orbital foreign body. Beyond the ocular sequelae is the enduring contraindication for performing magnetic resonance imaging in such a patient.

Numerous healthcare and surgical facilities impose blanket protocols mandating plain film radiographs of the surgical site be taken and interpreted by the surgeon and/or a radiologist should there be a miscount of suture needles used during any surgical procedure.

Completion of the surgical case may be delayed until radiologic confirmation that a suture needle is not present in the surgical field. However, the application of such policies when applied to ophthalmic surgical cases is questionable in light of the current literature.

According to the Patient Care Memorandum112-002-SAC, June 2006, of the Department of Veterans Affairs, Boston Healthcare System, VA, USA, 'surgical instruments must be counted, except for procedures that are routinely concluded with a radiograph (for example, an orthopaedic case to assure proper alignment of a bone or implant). In these cases, a radiograph is mandatory if an instrument count is not performed, and reading the radiograph to determine whether any instruments have been retained must be performed before the patient is transferred from the OR.'
'Department of

Ophthalmology, Veterans Affairs Boston Healthcare System, Boston, MA, USA

${ }^{2}$ Department of Ophthalmology, Boston University School of Medicine, Boston, MA, USA

${ }^{3}$ Department of Radiology, Veterans Affairs Boston Healthcare System, Boston, MA, USA

${ }^{4}$ Semphonic, Novato, CA, USA

Correspondence: MK Daly,

Department of Ophthalmology, VA Boston Healthcare System, 150 South Huntington Ave., Boston, MA 02130, USA Tel: + 857364 4635; Fax: + 8573644407 E-mail: Mary.Daly2@ va.gov

Received: 24 May 2008 Accepted in revised form: 19 September 2008 Published online: 17 October 2008

Presented as a poster at the annual meeting of the Association for Research in Vision and Ophthalmology, Fort Lauderdale, FL, USA, May 2007 


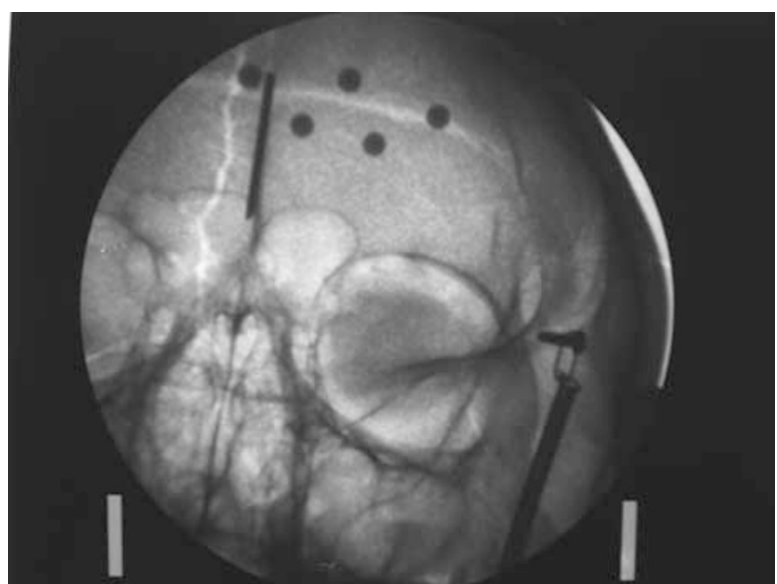

Figure 1 Portable plain film without a retained suture needle.

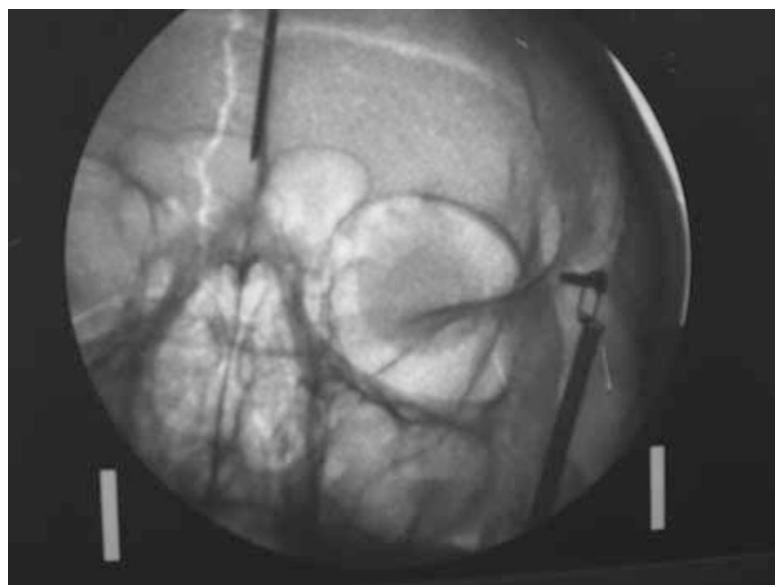

Figure 2 Portable plain film with a retained suture needle.

This study sought to evaluate the efficacy of plain film radiographs in reliably identifying retained suture needles used in ophthalmologic surgical cases.

\section{Materials and methods}

Ophthalmologic 10-0 nylon suture needles (Ethicon) were placed randomly into the conjunctival fornices, sclera, or cornea of 10 out of 20 porcine eyes. All eyes were placed within the orbit of a human cadaver skull, which was positioned on a soft tissue phantom. This allowed for the reproduction of the soft tissue and bony artefact normally present on radiographs taken from human patients.

Portable, plain film radiographs were taken of all eyes with a C-arm fluoroscopy machine (Figures 1 and 2). All radiographs were performed using radio-opaque markers to maintain a key, which could be used to identify films with a needle and those without. Control

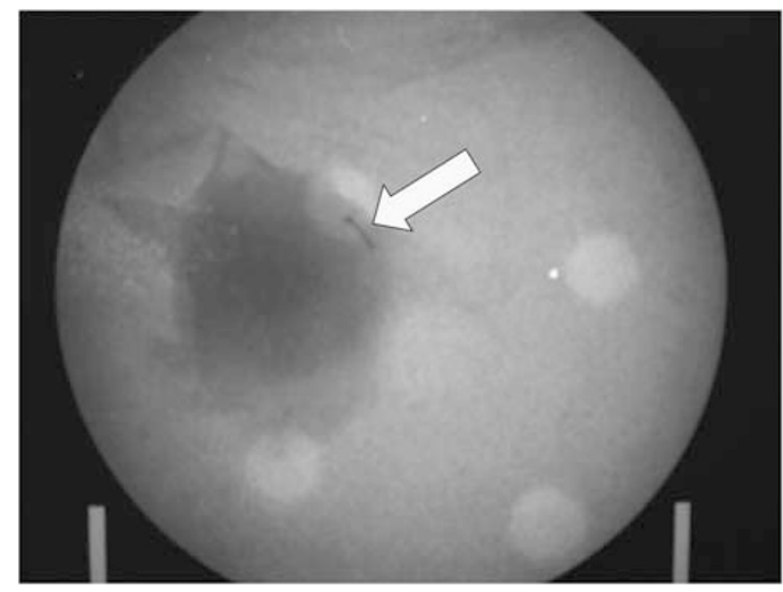

Figure 3 Control films identifying the presence of a needle (arrow).

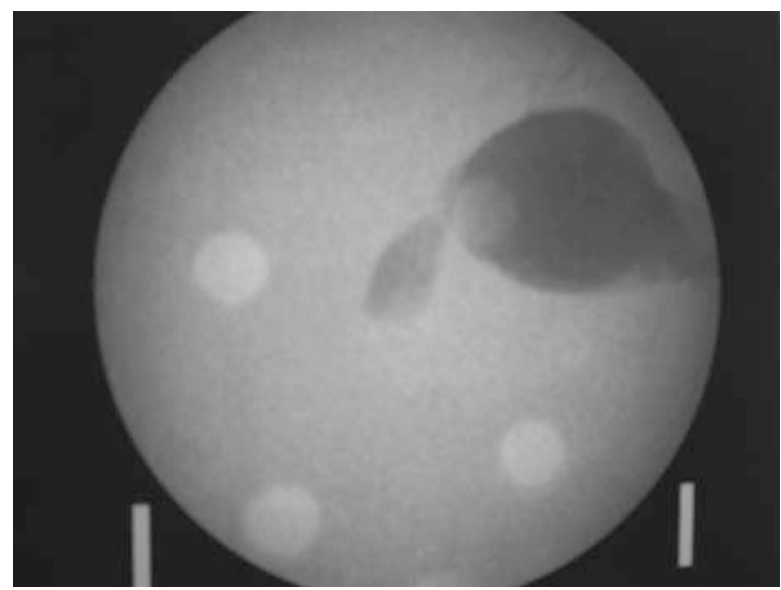

Figure 4 Control films identifying the absence of a needle.

films (Figures 3, and 4) of an eye with and without a suture needle were also taken to ensure the ability to accurately identify the presence of the suture needle in the absence of the skull and soft tissue artefact.

All films were then reviewed independently by six radiologists and six ophthalmologists who were asked to identify the presence or absence of a surgical needle. The readers were blinded to the experimental design, and no time limit was imposed. The sensitivity and specificity of identifying orbital foreign bodies by portable plain films was analysed for all subjects and further analysed on the basis of the subgroups of radiologists and ophthalmologists.

\section{Results}

Evaluation of the responses of all 12 reviewers showed a significant number of false-negative and false-positive responses. The overall sensitivity and specificity for all 
physicians reviewing radiographs with suspected intraoperative suture needles was 54 and $77 \%$, respectively.

Subgroup analysis showed that among radiologists reviewing the films, false-negative responses remained approximately the same with a sensitivity of $57 \%$. However, they did tend to have fewer false-positive responses, as demonstrated by a specificity of $87 \%$. On the other hand, ophthalmologists reviewing the films identified retained needles less reliably with a sensitivity of $51 \%$, and a specificity of $67 \%$.

\section{Discussion}

The value of plain film radiography in identifying intraocular foreign bodies has been a subject of significant controversy. During the advent of X-ray technology in the late 19th century, a landmark article found the technique to be a reliable test in indicating the mere presence of an intraocular foreign body; however, it was not consistent in accurately localizing the foreign body. ${ }^{1}$ Otto et $a l^{2}$ concluded that plain film radiographs can be used as an effective screening test for metallic foreign bodies in the orbit before MRI. However, their study did demonstrate a threshold size at which identification could only be achieved by CT scan and not by plain film.

Further complicating the matter, numerous case reports have been published in which plain film radiographs could not be relied upon to accurately identify intraocular foreign bodies. ${ }^{3-5}$ Bray and Griffiths ${ }^{6}$ found a low sensitivity and specificity, when screening for intraocular foreign bodies was performed by plain film radiographs. The presence of such clinical examples and the low sensitivities and specificities of plain film $X$-ray in identifying intraocular foreign bodies highlights the fact that such an imaging modality will not exclude any degree of certainty of the presence of an intraocular foreign body. As such, many have even suggested that clinical evaluation by an ophthalmologist may be a more effective means of evaluation. ${ }^{3}$

The general consensus throughout the literature is that the most effective means of evaluating the presence of soft tissue foreign bodies is through the use of CT scanning. ${ }^{4,7,8}$ Moreover, the use of ultrasound biomicroscopy has also been found by several investigators to be significantly better in identifying intraocular foreign bodies compared with X-ray. ${ }^{9-11}$ In fact, ultrasound has even been reported to detect intraocular foreign bodies in several instances when CT scanning failed to do so. ${ }^{10,11}$

To the best of our knowledge, this is the first study that we are aware of in which the efficacy of plain film radiographs as a diagnostic test for retained surgical needles was evaluated experimentally. We have shown a low sensitivity and specificity for both ophthalmologists and radiologists reviewing $\mathrm{X}$-rays used as a screening method for suspected retained suture needles. As we only used 10-0 nylon suture needles, extrapolation of these results to surgical cases in which larger bore needles are used should be performed cautiously in light of the aforementioned data by Otto et al. ${ }^{2}$ However, given the results in that study, which found that intraorbital steel wires of less than $0.12 \mathrm{~mm}^{3}$ were undetectable by plain film radiographs, it might be inferred that suture needles greater than $8-0$ would likewise be poorly identifiable.

The institution of generalized protocols mandating portable, plain film radiographs to be taken on every patient in the operating room when suture needle counts are inaccurate may not be appropriate in ophthalmologic surgical cases. In most ophthalmologic cases at this institution, inaccurate needle counts are on account of dropping of the needle during transfer from nurse to surgeon or surgeon to nurse and difficulty in locating the needle because of the small size. In those instances, the needle is not dropped near the surgical field. In most ophthalmic cases, with a small surgical field and microscopic wounds, it would be very difficult for a surgeon to lose a needle in the eye without being aware of the occurrence. The cost of such a mandate requiring the use of plain films, technician time, operating room personnel time, and radiological interpretation may not be the best way to handle inaccurate needle counts in ophthalmic surgical cases. It may be in the best interest of the patients and the institution to reform the X-ray policy in ophthalmic cases in which the needle count is inaccurate at the end of the case. It may be more effective to have a policy in eye cases, which mandates exploration of the surgical site by the ophthalmologist. If there is suspicion on the part of the surgeon that a needle or needle remnant is misplaced in the surgical site, a policy requiring CT scanning or ultrasound evaluation depending on the clinical cirmcumstance, may be more appropriate.

\section{Acknowledgements}

We thank the Massachusetts Lions Eye Research Fund for their assistance. This study was supported with resources from the Massachusetts Lions Eye Research Fund.

\section{References}

1 Kibbe $\mathrm{AB}$. The utility of the $\mathrm{X}$ rays in detecting and locating metallic particles in the eye 1897. Arch Ophthalmol 1996; 114: 1005. 
2 Otto PM, Otto RA, Virapongse C, Freidman S, Emerson S, $\mathrm{Li} \mathrm{KCP}$ et al. Screening test for detection of metallic foreign objects in the orbit before magnetic resonance imaging. Invest Radiol 1992; 27: 308-311.

3 Vote BJT, Simpson AJ. X-ray turns a blind eye to ferrous metal. Clin Experimental Ophthalmol 2001; 29(4): 262-264.

4 Reiner B, Seigel E, McLaurin T, Pomerantz S, ALlman R, Hebel JR et al. Evaluation of soft tissue foreign bodies: comparing conventional plain film radiography, computed radiography printed on film, and computed radiography displayed on a computer workstation. Am J Roentenol 1996; 167: 141-144.

5 Cooke CA, Mulholland DA. A closer look at anterior segment intraocular foreign bodies. Eye 2005; 19(4): 476-478.

6 Bray LC, Griffiths PG. The value of plain radiography in suspected intraocular foreign body. Eye 1991; 5: 751-754.

7 Williamson MR, Espinosa MC, Boutin RD, Orrison WW, Hart BL, Kelsey CA. Metallic foreign bodies in the orbits of patients undergoing MR imaging: prevalence and value of radiography and CT before MR. Am J Roentenol 1994; 162: 981-983.

8 Lagalla R, Manfre L, Caronia A, Bencivinni F, Duranti C, Ponte F. Plain film, CT and MRI sensibility in the evaluation of intraorbital foreign bodies in an in vitro model of the orbit and in pig eyes. Eur Radiol 2000; 10: 1338-1341.

9 Bryden FM, Pyott AA, Bailey M, McGhee CNJ. Real time ultrasound in the assessment of intraocular foreign bodies. Eye 1990; 4: 727-731.

10 Barash D, Goldenberg-Cohen N, Tzadok D, Lifshitz T, Yassur Y, Weinberger DOV. Ultrasound biomicroscopic detection of anterior ocular segment foreign body after trauma. Am J Ophthalmol 1998; 126: 197-202.

11 Deramo VA, Shah GK, Baumal CR, Fineman MS, Correa $\mathrm{ZM}$, Benson WE et al. Ultrasound biomicroscopy as a tool for detecting and localising occult foreign bodies after ocular trauma. Ophthalmology 1999; 106: 301-305. 